\title{
EDITORIAL
}

\section{Aims and Scope of JIDPS: Understanding, Improving, and Practicing of Design}

\author{
Yong Zeng ${ }^{*}$ \\ Concordia Institute for Information Systems Engineering, Concordia University, Montreal, Canada
}

As it is stated in its Aims and Scope, Journal of Integrated Design and Process Science (JIDPS) publishes research findings covering transdisciplinary notions of design and process with a focus on issues that deal with 1) understanding of design and process crossing boundaries of natural, human, and built environments, 2) principles, methods, and tools, and 3) applications of design and process science to engineering and social problems. The first issue is about the scientific foundation of design, which aims to understand the factors that have significant impact on design; the second issue is about the methodology and methods to improve the effectiveness and efficiency of design activities; and the last one is about the design of things following the guidance from the first two issues. This has formed the framework for the journal's editorial office to select and organize our publications. This issue of JIDPS includes three papers covering respectively these three aspects.

The first paper, titled "The nuances and management of multinational design teams - a Sino and Occidental comparison" by Waal et al., falls under the category of understanding design activities. It aims to identify key issues that affect the success in managing multinational design teams. Through interviewing designers of different nationality and design backgrounds about their experiences, it was found that background (cultural, national and economic) factors can affect how a designer approach a design problem, particularly for framing and goal analysis. These individual background factors determine how a designer may work in a team with designers from another background. This research noted: 1) designers with a low level attitude toward design and a lower level of emotional intelligence tend to be less effective to work in such teams; 2) the introduction of information technology tools may increase the chance of success thanks to the increase of shared understanding of the design; 3) through the standardisation of design practices, background differences would be less significant; and 4) the impact of background differences on design skills and practice does not appear to be permanent.

The second paper, titled "A software quality model by using discriminant analysis predictive technique" by Ronchieri et al., falls under the category of methods to improve design effectiveness through enhanced quality and reduced errors. It aims to periodically predict the quality of software code development at any stage and to identify problems of software development at an early phase. In order to achieve this objective, the authors extend a mathematical model that connects software best practices with a set of metrics using a risk-threshold-based discriminant analysis technique. The extended model was tested using the data gathered from several European Middleware Initiative packages, which have different scopes and characteristics. Such a model is highly expected of conceptual design and strategic planning processes.

\footnotetext{
* Corresponding author. Email: zeng@ ciise.concordia.ca. Tel: (+1)514-8482424 ext. 5801.
} 
The third paper, titled "An integrated computer program for blade design of small to medium wind turbine generators" by Huang et al., falls into the category of design practices. Following a systematic analysis of design requirements, this paper introduces an integrated computer aided blade design system that the authors developed for small to medium wind turbine generators. From the Environment-Based Design point of view (Zeng, 2011), the environment of the developed computer program includes blade designers, commercial CAD software systems, the blade models under construction, the aerodynamic performance analysis of blades, and stress analysis of the blades. The software thus aims to reduce the iterations by simplifying the recursive dependence between the modification of blade model and the performance analysis. The developed system is able to improve the efficiency of blade design through a combination of aerodynamic and structural considerations.

As the JIDPS is going into its fourth year after it took a new format and new structure, we welcome more submissions covering the understanding, improving, and practicing of design, which can be engineering, economic, or social. We particularly encourage a transdisciplinary approach to these various kinds of design problems.

\section{References}

Zeng, Y. (2011). Environment-Based Design (EBD). ASME Conference Proceedings, 2011(54860), 237250.

\section{Author Biography}

Dr. Yong Zeng is a professor in the Concordia Institute for Information Systems Engineering at Concordia University, Montreal, Canada. Zeng's research interest is in understanding and improving design activities, especially creative design activities. In addition to developing a new design methodology Environment Based Design (EBD), he has been developing formal and experimental approaches to design research. He collaborates with aerospace companies, pharmaceutical companies, software development companies, and municipality for the applications of his research results. 